\title{
DIGITALIZAÇÃO DO CAMPO A FAVOR DA PRODUÇÃO DE SOJA E DA AGRICULTURA NO BRASIL E NO MUNDO
}

\author{
DIGITIZATION OF THE FIELD IN FAVOR OF SOYBEAN PRODUCTION AND \\ AGRICULTURE IN BRAZIL AND IN THE WORLD
}

\begin{abstract}
Samuel Cardoso Ferreira - samukajettatsi@icloud.com Faculdade de Tecnologia de Taquaritinga - Taquaritinga - São Paulo - Brasil

Fábio Alexandre Cavichioli - fabio.cavichioli@fatectq.edu.br Faculdade de Tecnologia de Taquaritinga - Taquaritinga - São Paulo - Brasil

DOI: 10.31510/infa.v18i1.1166

Data de submissão: 17/04/2021

Data do aceite: 09/07/2021

Data da publicação: 30/07/2021
\end{abstract}

\section{RESUMO}

O Brasil é o maior produtor de soja do mundo, sendo o estado do Mato Grosso (MT) o principal produtor de soja brasileiro com 35,885 milhões de toneladas produzidas, seguido por Paraná (PR) 21,598 milhões de toneladas, Rio Grande do Sul (RS) que produz em torno de 11,444 milhões de toneladas. A oleaginosa é a principal cultura cultivada e representa cerca de $50 \%$ da colheita de grãos no Brasil, estimada em 264,8 milhões de toneladas. O objetivo deste trabalho é demonstrar a importância da digitalização do campo, a utilização da internet das coisas ou simplesmente IoT e big data. Os resultados apontam que é de suma importância para o produtor rural ter em mãos dados concretos e assertivos que a tecnologia pode proporcionar. A obtenção destes dados possibilitam redução no custo operacional, aplicação de insumos as taxas variáveis e previsibilidade de ações por causa de análise de históricos realizadas com a big data. Este trabalho foi realizado através de levantamentos bibliográficos de artigos, livros, trabalhos acadêmicos e estudos de caso.

Palavras-chave: Agronegócio. Agricultura digital. Tecnologia da informação. Produtividade

\begin{abstract}
Brazil is the largest producer of soybeans in the world, the state of Mato Grosso (MT) being the main producer of Brazilian soybeans with 35,885 million tons produced, followed by Paraná (PR) 21,598 million tons, Rio Grande do Sul (RS) ) which produces around 11,444 million tonnes. Oilseeds are the main cultivated crop and represent about $50 \%$ of the grain harvest in Brazil, estimated at 264.8 million tons. The objective of this work is to demonstrate the importance of digitalizing the field, the use of the internet of things or simply IoT and big data. The results show that it is extremely important for the rural producer to have in hand concrete and assertive data that technology can provide. Obtaining this data makes it possible to reduce operating costs, apply data such as variable rates and predictability of actions due to the analysis
\end{abstract}


of records made with big data. This work was carried out through bibliographic surveys of articles, books, academic works and case studies.

Keywords: Agribusiness. Digital agriculture. Information Technology. Productivity

\section{INTRODUÇÃO}

A soja é uma planta originária da China. Seu nome científico é Glycine max L.; faz parte da família Fabaceae (leguminosas), assim como a ervilha, o feijão e a lentilha (JARDINE \& BARROS, 2021).

Embora haja registros históricos que apontam para cultivos experimentais de soja na Bahia já em 1882, a introdução da soja no Brasil tem o ano de 1901 como marco principal: é quando começam os cultivos na Estação Agropecuária de Campinas e a distribuição de sementes para produtores paulistas. O grão começa a ser mais facilmente encontrado no País a partir da intensificação da migração japonesa, nos anos 1908. Em 1914, é oficialmente introduzida no Rio Grande do Sul - estado que apresenta condições climáticas similares às das regiões produtoras nos Estados Unidos (APROSOJA, 2021).

O Brasil é um dos maiores produtores de soja do mundo. Parte disso se deve aos avanços tecnológicos nas lavouras, que têm auxiliado a otimizar a cultura do grão. A produção de soja no Brasil na safra 2019/20 foi de 124,845 milhões de toneladas, sendo o estado do Mato Grosso o maior produtor de soja no país com 35,885 milhões de toneladas produzidas, segundo a CONAB (2020).

A inovação no campo é marcada pela introdução de tecnologias, desde o uso dos primeiros maquinários agrícolas, passando pela revolução verde e a agricultura de precisão, com sensores inteligentes e colheitadeiras automáticas (PEREIRA, 2018).

A mudança tecnológica na agricultura nos últimos anos tem sido o foco da pesquisa na área, por desempenhar um papel decisivo no atendimento às demandas futuras de produtos agrícolas (Dietrich, Schmitz, Lotze-Campen, Popp, \& Müller, 2014; Hertel, Baldos, \& Mensbrugghe, 2016). O ambiente tecnológico de rápidas mudanças tende a influenciar os pequenos agricultores e estes, às vezes, enfrentam dificuldades para assimilar a tecnologia e os modelos de negócio do sistema econômico. Apesar das dificuldades, a inovação na agricultura tem mostrado uma forte correlação com o aumento da produção agrícola (Runge et al., 2003). O incremento da produção agrícola tem sido o resultado da mudança tecnológica induzida pelos investimentos em pesquisa e desenvolvimento (P\&D) (Dietrich et al., 2014). 
O presente trabalho tem por objetivo apresentar a importância da digitalização do campo e as vantagens para a produção de soja no Brasil.

\section{FUNDAMENTAÇÃO TEÓRICA}

\subsection{A cultura de soja}

A soja é uma planta originária da China. Seu nome científico é Glycine max L.; faz parte da família Fabaceae (leguminosas), assim como a ervilha, o feijão e a lentilha (JARDINE \& BARROS, 2021).

As primeiras citações do grão aparecem no período entre 2883 e 2838 AC, quando a soja era considerada um grão sagrado, ao lado do arroz, do trigo, da cevada e do milheto. Um dos primeiros registros do grão está no livro "Pen Ts'ao Kong Mu", que descrevia as plantas da China ao Imperador Sheng-Nung. Para alguns autores, as referências à soja são ainda mais antigas, remetendo ao "Livro de Odes", publicado em chinês arcaico (EMBRAPA, 2021).

O aumento do plantio de soja no Brasil sempre esteve associado ao desenvolvimento rápido de tecnologias e pesquisas focadas no atendimento da demanda externa. Tanto que em meados dos anos 70 a cultivar já era a principal cultura do agronegócio nacional: a produção havia passado do 1,5 milhão de toneladas em 1970 para mais de 15 milhões de toneladas em 1979. Importante notar que essa ampliação desde esse início esteve intrinsecamente ligada aos investimentos no aumento de produtividade, e não necessariamente de área (que de 1,3 milhão de hectares passou para 8,8 milhões de hectares na década). Os índices de produtividade nesse período saíram do patamar de 1,14 t/ha para 1,73 t/ha. (APROSOJA, 2021).

A explosão do preço da soja no mercado mundial, em meados de 1970, desperta ainda mais os agricultores e o próprio governo brasileiro. O País se beneficia de uma vantagem competitiva em relação aos outros países produtores: o escoamento da safra brasileira ocorre na entressafra americana, quando os preços atingem as maiores cotações. Desde então, o país passou a investir em tecnologia para adaptação da cultura às condições brasileiras, processo liderado pela Empresa Brasileira de Pesquisa Agropecuária (EMBRAPA, 2021).

A soja começou a ser cultivada no Rio Grande do Sul, como uma opção de rotação com o trigo. Depois de assentada nesse estado, expandiu-se para o norte, chegando a Santa Catarina, Paraná e São Paulo (MUELLER et al, 2002). 


\title{
2.2 Produção de soja no Brasil
}

Com um aumento de área em 3,4\%, a produção de soja na safra 2020/21 pode chegar a 133,7 milhões de toneladas no país. A oleaginosa é a principal cultura cultivada e representa cerca de 50\% da colheita de grãos no Brasil, estimada em 264,8 milhões de toneladas (CONAB, 2021).

O estado do Mato Grosso (MT) é o principal produtor de soja no Brasil com 35,885 milhões de toneladas produzidas, seguido por Paraná (PR) 21,598 milhões de toneladas, Rio Grande do Sul (RS) que produz em torno de 11,444 milhões de toneladas (CONAB, 2021).

\subsection{Digitalização do campo, internet das coisas (IoT) e big data}

Segundo FIELDVIEW (2020), nas últimas 4 décadas, a produção da agricultura brasileira registrou aumento de $386 \%$. Em contrapartida, a área ocupada pelo setor subiu apenas $33 \%$. Isso significa que o país produziu mais sem aumentar, na mesma proporção, a área plantada. O motivo para esse crescimento tem nome: desenvolvimento tecnológico.

\begin{abstract}
Diante de todos esses desafios apresentados na agricultura, principalmente o de aumentar a produção agrícola sem ampliar significativamente a área plantada, tornase premente o uso cada vez mais intenso de novas tecnologias para permitir os ganhos de produtividade de forma sustentável. Nesse contexto surgiu um novo fator de produção que está modificando a base de crescimento econômico para os países em todo o mundo. Trata-se da transformação digital, uma nova abordagem em que as Tecnologias de Informação e Comunicação (TIC) desempenham papel-chave na transformação da estratégia, da estrutura, da cultura e dos processos das organizações, utilizando o alcance e o poder da internet (MASSRUHÁ et al. 2020).
\end{abstract}

De acordo com ASSAD \& PANCETTI (2009), as tecnologias de informação e comunicação (TICs) estão inseridas nas atividades rurais como fator de competitividade. Os controles agronômicos, zootécnicos e administrativos utilizam softwares os mais variados. A integração entre os agentes da cadeia de valor com o mercado, tanto para a comercialização de produtos agrícolas, como para a captação das necessidades dos consumidores e rastreamento de mercadorias, passa, progressivamente, a utilizar o formato digital.

O IoT e a utilização do "big data" poderão ser utilizados por diferentes segmentos do agronegócio. A tecnologia de uso mais imediato são os sensores sem fio, localizados no solo ou em tratores, o que - em conjunto com o uso de poderosos softwares de "big data" - permitirá um mapeamento de campo muito preciso e, plantio personalizado. Drones e satélites ainda são uma ferramenta mais dispendiosa, mas os preços certamente cairão com a expansão do setor (SEIXAS \& CONTINI, 2017). 
O termo Internet das Coisas, ou Internet of Things (IoT) em inglês, foi apresentado primeiramente por Kevin Ashton da MIT Auto Centre, em uma apresentação sobre RFID e a cadeia de suprimentos de uma grande companhia, em 1999. (Ashton, 2009).

Existem outras aplicações da IoT na agricultura, por exemplo:

- Identificação da necessidade de correção do solo;

- Identificação por demanda de irrigação;

- Diagnostico da lavoura com uso de drones;

- Implantação de estufas inteligentes;

- $\quad$ Controle de pragas na lavoura;

A evolução tecnológica nas últimas décadas aumentou significativamente o volume de dados disponíveis para as organizações, impactando seus processos decisórios e resultados. Sistemas relacionados ao Big Data têm adquirido cada vez mais importância nos negócios nos últimos anos, pois as organizações necessitam cada vez mais analisar dados abundantes e variados (Chen, Chiang \& Storey, 2012).

Benefícios esperados do uso do Big Data incluem integração de dados de diversas fontes, captura e análise de grandes volumes de dados estruturados e não estruturados, melhora das capacitações analíticas gerais, análise de informação em tempo real, redução de custos da análise e processamento de dados, maior transparência das informações utilizadas para a tomada de decisões, personalização mais ágil de produtos e serviços e criação de novos modelos de negócios (Davenport, Barth \& Bean, 2012).

O Big Data na agricultura trará ganhos em sustentabilidade. A modelagem agronômica permite antecipar as condições ideais para a ocorrência de uma doença levando em conta, por exemplo, a presença do patógeno, o nível de umidade e a temperatura ao longo de um número de dias. Os dados são usados para simular a probabilidade de a doença atacar a plantação, permitindo ao agricultor embasar a decisão de quando pulverizar - e também o volume adequado de inseticida ou fungicida a ser aplicado na lavoura (LEUZINGER, 2016).

\subsection{Barreiras da digitalização do campo}

Quando o assunto é expansão da digitalização no campo, a principal barreira ainda é a conectividade. A extensa dimensão territorial do Brasil, a falta de infraestrutura nas zonas rurais e o alto investimento, fator que inviabiliza principalmente pequenos e médios 
produtores de terem acesso à internet, ainda permanecem sendo os principais impeditivos dessa expansão (GALINARI, 2020).

\section{PROCEDIMENTOS METODOLÓGICOS}

Serão realizadas pesquisas em livros, artigos científicos e periódicos, que de acordo com Gil (2008) caracteriza-se como a pesquisa bibliográfica. Koche (1997) mostra que a pesquisa bibliográfica pode ser utilizada neste caso, pois busca-se ampliar o grau de conhecimentos em uma determinada área, compreendendo ou delimitando melhor o problema de pesquisa, bem como descrever o estado da arte. Para Gil (2008), pesquisa bibliográfica é desenvolvida com base em material já elaborado, constituído principalmente de livros e artigos científicos.

\section{RESULTADOS E DISCUSSÃO}

Neste estudo foi verificado números da produção de soja no Brasil desde a sua introdução até o seu crescimento exponencial em meados dos anos 70. Também foram discutidos sobre a digitalização do campo e a importância das novas tecnologias quando empregadas de forma correta e seus benefícios.

Atualmente existem pesquisas em consorcio com brasileiros e europeus onde estão instalando sensores e construindo uma plataforma computacional para a gestão inteligente da irrigação de precisão, baseada em internet das coisas (IoT, na sigla em inglês). Os testes localizados no Brasil, Espanha e Itália são conduzidos pela pesquisa internacional Smart Water Management Platform.

A plataforma, baseada em tecnologia de ponta, integra uma rede de sensores sem fio
que coletam dados agronômicos e de umidade do solo, em diferentes áreas e
profundidades, e avaliam, por exemplo, a necessidade de irrigação das plantas. As
informações são enviadas para computadores instalados nas propriedades agrícolas e
de lá seguem para uma nuvem computacional, onde são armazenadas e processadas,
com o objetivo de otimizar o processo de irrigação e o uso da água na agricultura
(SILVA, 2020)

Segundo SILVA (2020) Os primeiros experimentos já demonstraram a viabilidade e o potencial de reprodução do sistema no Brasil e no exterior. Para validar a tecnologia e analisar seu desempenho e escalabilidade, também houve uma simulação do uso de até 45 mil sensores para coleta de dados no campo e integração com outros modelos computacionais. Testes realizados nos três países apontaram, no entanto, a necessidade de modelos personalizados para 
o uso racional da água e a integração de diferentes tecnologias para que a aplicação da IoT seja adotada de forma generalizada na irrigação de precisão.

Pesquisadores utilizaram big data para compreender melhor os efeitos dos eventos climáticos conhecidos como El Niño e La Niña na variabilidade da distribuição das chuvas e a consequente interferência no cultivo de grãos no Brasil. O estudo analisou e cruzou dados de 50 municípios nos estados de Rondônia, Mato Grosso, Goiás e Tocantins, responsáveis por $39 \%$ da produção de grãos no País, e resultou em um calendário de cultivo para diminuir riscos de estresses hídricos associados à perda de produção nessas localidades (PEIXOTO, 2020).

O mesmo autor PEIXOTO (2020) diz que um dos resultados foi a elaboração de um calendário de cultivo para diminuir riscos de estresses hídricos associados à perda de produção nas localidades estudadas.

\section{CONSIDERAÇÕES FINAIS}

A partir do levantamento realizado pelas pesquisas bibliográficas, observa-se que o Brasil é sem sombra de dúvidas o maior produtor de soja do mundo. Observou-se também o quanto é importante o advento da tecnologia no campo.

A $I o T$ é considerada uma realidade para agricultura nos próximos anos pois os seus benefícios são incontáveis.

Este trabalho é útil pois demonstra tecnologias existente que podem propiciar melhorarias na tomada de decisão de agricultores, governos, seguradoras, indústrias de insumos e outros segmentos envolvidos na cadeia da soja.

\section{REFERÊNCIAS}

APROSOJA. ASSOCIAÇÃO DOS PRODUTORES DE SOJA E MILHO DE MATO GROSSO. A história da soja. Cuiabá. 2021. Disponível em: <http://www.aprosoja.com.br/sojae-milho/a-historia-da-soja>. Acesso em: 16 abr. 21.

Ashton, Kevin. That 'Internet of Things' thing. Publicano no RFID Journal, 2009. Acesso em 17 abr. 21

Chen, H., Chiang, R. H. L., \& Storey, V. C. (2012). Business intelligence and analytics: From big data to big impact. Mis Quarterly, 36(4), 1-22. 
CONAB. COMPANHIA NACIONAL DE ABASTECIMENTO. Acompanhamento de safra brasileiro - grãos: Nono levantamento - safra 2019/2020.: Brasília: Companhia Nacional de Abastecimento. 2020.

CONAB. COMPANHIA NACIONAL DE ABASTECIMENTO. Acompanhamento de safra brasileiro - grãos: safra 2020/2021.: Brasília: Companhia Nacional de Abastecimento. 2021.

Davenport, T., Barth, P., \& Bean, R. (2012). How 'Big data' is different. MIT Sloan Management Review, 54(1), 43-46.

DIETRICH, J. P., SCHMITZ, C., Lotze-Campen, H., Popp, A., \& Müller, C. (2014). Forecasting technological change in agriculture-an endogenous implementation in a global land use model. Technological Forecasting and Social Change, 81, 236-249.

EMBRAPA. EMPRESA BRASILEIRA DE PESQUISA AGROPECUÁRIA. História da soja. Brasília. 2021. Disponível em: $<$ https://www.embrapa.br/web/portal/soja/cultivos/soja1/historia $>$. Acesso em: 16 abr. 21.

FIELDVIEW, CLIMATE FIELD VIEW. A digitalização do campo pode ser caminho para uma agricultura mais sustentável. 2020. Disponível em: <https://blog.climatefieldview.com.br/adigitalizacao-do-campo-pode-ser-caminho-para-uma-agricultura-mais-sustentavel $>$. Acesso em: 13 abr. 21.

GALINARI, G. Pesquisa mostra o retrato da agricultura digital brasileira. EMBRAPA Informática. 2020. Brasília. Disponível em: https://www.embrapa.br/busca-de-noticias//noticia/54770717/pesquisa-mostra-o-retrato-da-agricultura-digital-brasileira. Acesso em: 17 abr. 21.

GIL, Antonio Carlos. Como elaborar projetos de pesquisa. 4. ed. São Paulo: Atlas, 2008.

Hertel, T. W., Baldos, U. L. C., \& van der Mensbrugghe, D. (2016). Predicting long-term food demand, cropland use, and prices. Annual Review of Resource Economics, 8, 417-441.

JARDine, J. G. BARRos, T. D. Soja. AGEITEC. Agência Embrapa de Tecnologia da Informação. Brasilia. 2021. Disponível em: $<$ https://www.agencia.cnptia.embrapa.br/gestor/agroenergia/arvore/CONT000fbl23vmz02wx 5eo0sawqe3vtdl7vi.html>. Acesso em: 16 abr. 21.

LEUZINGER, B. Agricultura digital e big data - a nova revolução na lavoura. 2016. Disponível em: $\quad<$ https://www.projetodraft.com/agricultura-digital-e-big-data-a-nova-revolucao-nalavoura/>. Acesso em: 15 abr. 21.

MUELLER, C. et al. Análise da expansão da soja no Brasil. Brasília: Banco Mundial, 2002. [On line]. Acesso em: 14 abr. 21

PEIXOTO, R. Ciência usa big data para reduzir riscos climáticos na produção de grãos no Brasil. EMBRAPA. Brasília, 2020. Disponível em: <https://www.embrapa.br/busca-de- 
noticias/-/noticia/56525505/ciencia-usa-big-data-para-reduzir-riscos-climaticos-na-producaode-graos-no-brasil>. Acesso em: 14 abr. 21.

PEREIRA, N. R. Digitalização do campo busca transformar agricultura. Brasília. EMBRAPA, 2018. Disponível em: <https://www.embrapa.br/busca-de-noticias//noticia/36548966/digitalizacao-no-campo-busca-transformar-agricultura $>$. Acesso em: 16 abr. 21

Runge, C. F., Senauer, B., Pardey, P. G., \& Rosegrant, M. W. (2003). Ending hunger in our lifetime: Food security and globalization. London: International Food Policy Research Institute.

SEIXAS, M. A. CONTINI, E. Internet das coisas (IoT) - Inovação para o agronegócio. Brasília, novembro, 2017

SILVA, J. Internet das coisas é testada em irrigação de precisão. EMBRAPA. Brasília, 2020. Disponível em: <https://www.embrapa.br/busca-de-noticias/-/noticia/49976152/internet-dascoisas-e-testada-em-irrigacao-de-precisao>. Acesso em: 15 abr. 2021. 